\title{
BMJ Open Experiences of pregnant women with gestational diabetes mellitus: a systematic review of qualitative evidence protocol
}

\author{
Jing He (1) , ${ }^{1}$ Yuchen Wang (D) , ${ }^{1}$ Yanqun Liu, ${ }^{1}$ Xiaoli Chen (D) , Jinbing Bai ${ }^{2}$
}

To cite: He J, Wang $Y$, Liu Y, et al. Experiences of pregnant women with gestational diabetes mellitus: a systematic review of qualitative evidence protocol. BMJ Open 2020;10:e034126. doi:10.1136/ bmjopen-2019-034126

- Prepublication history and additional material for this paper are available online. To view these files, please visit the journal online (http://dx.doi. org/10.1136/bmjopen-2019034126).

JH and YW contributed equally.

Received 08 September 2019 Revised 19 December 2019 Accepted 20 December 2019

Check for updates

(C) Author(s) (or their employer(s)) 2020. Re-use permitted under CC BY-NC. No commercial re-use. See rights and permissions. Published by BMJ.

${ }^{1}$ School of Health and Sciences, Wuhan University, Wuhan, China ${ }^{2}$ Nell Hodgson Woodruff School of Nursing, Emory University, Atlanta, Georgia, USA

\section{Correspondence to}

Associate Professor Yanqun Liu; liuyanqun1984@163.com and Associate Professor Xiaoli Chen; chenx|201313@163.com

\section{ABSTRACT}

Introduction The incidence of gestational diabetes mellitus (GDM) is increasing and an issue of global concern. GDM can cause severe adverse effects for pregnant women and their fetuses. This systematic review is proposed to explore women's experiences during the pregnancy with GDM. This review will provide insights into the physical, psychological and social adaptation experiences of women with GDM that can help to identify challenges of glycaemic control and provide targeted care and interventions to improve maternal and child health. Methods and analysis The databases we will search include English databases (ie, PubMed, CINAHL, Embase, the Cochrane Library, Web of Science, Joanna Briggs Institute (JBI) Database of Systematic Reviews, PsycINF0, OpenGrey and Deep Blue) and Chinese databases (ie, China Biology Medicine disc, China National Knowledge Infrastructure, and VIP Database for Chinese Technical Periodicals). Published qualitative evidence of life changes or experiences of the women with GDM will be searched. There will be no limits on publication year. Two reviewers will independently use the JBI Critical Appraisal Checklist for Qualitative Research for methodological validity prior to inclusion in this review. Any disagreements regarding article evaluation will be resolved through discussion or with a third reviewer. Data will be extracted using the standardised data extraction tool from JBI System for the Unified Management, Assessment and Review of Information. Synthesis will include in-depth reading of the original text and the discovery of the results, and then summarising similar categories for more advanced synthesised findings. The final synthesised findings will be graded according to the ConQual approach for establishing confidence.

Ethics and dissemination This study does not require ethical approval as primary data will not be collected. Results of this systematic review will be submitted to peer-reviewed international journals for publication and be presented in relevant international conferences.

PROSPERO registration number CRD42019132065.

\section{INTRODUCTION}

During the pregnancy, the body is resistant to insulin in response to physiological changes, which may lead to a high risk of gestational diabetes mellitus (GDM) for some pregnant
Strengths and limitations of this study

- There is an urgent need to synthesise qualitative evidence about gestational diabetes mellitus (GDM) women's experiences from diagnosis to the end of childbirth so that we can provide insights into GDM management and interventions.

- Results of this review will identify strengths and weaknesses of the current literature regarding GDM women's experiences.

- Systematic review of qualitative empirical evidence from multiple regions and cultures will facilitate the dissemination of findings regarding GDM and promote nursing practice in GDM.

- This review will not analyse the experiences of postpartum women.

women. ${ }^{1}$ GDM is defined as the first abnormal glucose metabolism during the pregnancy. ${ }^{2}$ Prevalence of GDM is influenced by screening methods, diagnostic criteria and the inherent characteristics in every study population, making it difficult to estimate global prevalence, but the prevalence of GDM poses significant challenges to global public health. ${ }^{3}$ Using IADPSG (International Association of Diabetes and Pregnancy Study Groups) diagnostic criteria, the study found that the incidence of GDM fluctuated between $5.12 \%$ and $33.3 \%$ in mainland China. ${ }^{4}$ The primary risk factors for GDM include maternal age, race/ ethnicity, parity, body mass index, hypertension and smoking status. ${ }^{5}$

As there are few or no symptoms, pregnant women are usually not aware of the GDM until it is diagnosed at a routine prenatal screening. ${ }^{6}$ Compared with healthy pregnant women, many serious pregnancy complications are associated with GDM. GDM has serious adverse effects on the health of both the mother and the infant. GDM can directly lead to caesarean section, and many other complications such as hypertension, abortion 


\begin{tabular}{|c|c|c|c|c|c|c|c|c|c|c|c|c|c|}
\hline & & & Summa & ry JBI Qu & alitative $A$ & Assessme & nt and $R$ & eview In & strumer & t (QARI) & & & \\
\hline \begin{tabular}{|l} 
\\
Record \\
details/ \\
Full \\
reference
\end{tabular} & $\begin{array}{l}\text { Is there } \\
\text { congruity } \\
\text { between the } \\
\text { stated } \\
\text { philosophical } \\
\text { perspective } \\
\text { and the } \\
\text { research } \\
\text { guestion or } \\
\text { methodology? }\end{array}$ & $\begin{array}{l}\text { Is there } \\
\text { congruity } \\
\text { between the } \\
\text { research } \\
\text { methodology } \\
\text { and the } \\
\text { research } \\
\text { guestion or } \\
\text { pbjectives? }\end{array}$ & $\begin{array}{l}\text { Is there } \\
\text { congruity } \\
\text { betiveen the } \\
\text { research } \\
\text { methodology } \\
\text { and the } \\
\text { methods } \\
\text { used to } \\
\text { collect data? }\end{array}$ & $\begin{array}{l}\text { Is there } \\
\text { congruity } \\
\text { between the } \\
\text { research } \\
\text { methodology } \\
\text { and the } \\
\text { representation } \\
\text { and analysis o } \\
\text { data? }\end{array}$ & $\begin{array}{l}\text { Is there } \\
\text { congruity } \\
\text { between the } \\
\text { research } \\
\text { methodology } \\
\text { and the } \\
\text { interpretation } \\
\text { fof results? }\end{array}$ & $\begin{array}{l}\text { Is there a } \\
\text { statement } \\
\text { locating the } \\
\text { researcher } \\
\text { culturally or } \\
\text { theoretically? }\end{array}$ & $\begin{array}{l}\text { Is the } \\
\text { influence o } \\
\text { the } \\
\text { researcher } \\
\text { on the } \\
\text { research, } \\
\text { and vive- } \\
\text { versa, } \\
\text { addressed? }\end{array}$ & $\begin{array}{l}\text { Are } \\
\text { participants } \\
\text { and their } \\
\text { oides } \\
\text { adequately } \\
\text { everesented }\end{array}$ & 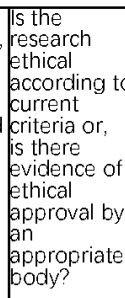 & 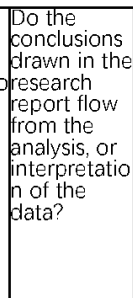 & score & $\begin{array}{l}\text { leek } \\
\text { further } \\
\text { into }\end{array}$ & $\begin{array}{l}\text { Comments } \\
\text { (including } \\
\text { reason for } \\
\text { exclusion) }\end{array}$ \\
\hline & Q1 & Q2 & Q3 & Q4 & Q5 & Q6 & Q7 & Q8 & Q9 & Q10 & $/ 10$ & & \\
\hline & & & & & & & & & & & & & \\
\hline & & & & & & & & & & & & & \\
\hline
\end{tabular}

Figure 1 Joanna Briggs Institute quality appraisal tool.

and fetal malformation. ${ }^{78}$ For the fetus, high risks of complications of GDM include fetal intrauterine growth retardation and macrosomia. ${ }^{9}$ Further more, studies also showed that GDM had long-term negative effects in offspring, such as high risk of type $2 \mathrm{DM}$ (T2DM), obesity and cardiovascular diseases. ${ }^{10-13}$ Additionally, exposure to maternal GDM is an independent risk factor for long-term neuropsychiatric morbidity in the offspring. ${ }^{14}$ Women with GDM have seven-fold increased risk for T2DM compared with normal pregnant women. ${ }^{15} 16$ There are $35 \%-50 \%$ of women with GDM may have recurrence in subsequent pregnancies. ${ }^{17}$

Due to negative consequences of GDM to pregnant women and infants, intervention programmes on GDM treatments have been developed to improve maternal and newborn health outcomes. ${ }^{18}$ Current interventions primarily include glucose monitoring dietary, physical activity, pharmacological hypoglycaemic agents (oral hypoglycaemic agents or insulin), health education, psychological and selective combine intervention. ${ }^{19}$ Many studies have been published on the issue of diagnosis, testing and treatment of GDM. However, the experiences of women diagnosed with GDM during treatment are still not well studied. ${ }^{20}$ GDM is associated with lifestyle changes and emotional reactions due to treatment. ${ }^{21}$ Information about the diagnosis may be distressing to pregnant women. ${ }^{223}$ Pregnant women may receive certain types of health-related guidance from medical institutions after the diagnosis of GDM. However, how they accept and manage the required behavioural changes needs to be further studied. ${ }^{24}$ For instance, many women with GDM perceive family guidance as a responsibility and also lack support from family. An inadequate social and family support can create barriers to behavioural changes and social isolation, and therefore, these healthy behavioural changes will be difficult to be maintained..$^{25} 26$

Women who suffer from GDM may have distressing experiences such as self-accusation and anxiety for the new baby. Besides, women may change their lifestyles to manage pregnant blood glucose. ${ }^{27-29}$ But there are also a large number of women who are not trying to change their behaviours. ${ }^{30}$ Quantitative studies related to GDM have provided some clinical guidance, such as the need for dietary management to effectively control blood glucose. ${ }^{31}{ }^{32}$ However, there are many challenges in the process of self-management, and it is very difficult for women to maintain a reasonable blood glucose level. ${ }^{33}$

The purpose of this review is to gain a deeper understanding regarding diagnosis and knowledge of the disease of pregnant women with GDM, pregnant women's beliefs about health, expectations of pregnancy outcomes and maternal and child's future health, as well as challenges and needs during pregnancy. This review will provide insights into the physical, psychological

\begin{tabular}{|c|c|c|c|c|c|c|c|c|c|c|}
\hline \multicolumn{11}{|c|}{ Modified JBI Qualitative data extraction tool } \\
\hline $\begin{array}{c}\text { Study } \\
\text { (Name and } \\
\text { Authors) }\end{array}$ & $\begin{array}{c}\text { Phenomen } \\
\text { a of } \\
\text { interest }\end{array}$ & Methodology & ylMethods & Setting & Geographical & | Cultural & $\begin{array}{c}\text { Participants } \\
\text { (Age, } \\
\text { relevant } \\
\text { number, } \\
\text { sample) }\end{array}$ & $\begin{array}{c}\text { Data } \\
\text { analysis }\end{array}$ & \begin{tabular}{|c|} 
Authors \\
Conclusion
\end{tabular} & Comments \\
\hline & & & & & & & & & & \\
\hline & & & & & & & & & & \\
\hline
\end{tabular}

Figure 2 Data extraction tool to include all the results and findings sections of each included study. JBI, Joanna Briggs Institute. 
Systematic review title: the experiences of the pregnant with gestational diabetes: a systematic review of qualitative evidence

Population: persons who have been diagnosed with GDN during pregnancy

Phenomena of interest: the exposition of the experiences of women with GDM during pregnancy

Context: in the experiences and feelings of women with GDM during pregnancy

\begin{tabular}{|l|l|l|l|l|}
\hline Synthesized finding & $\begin{array}{l}\text { Type of } \\
\text { research }\end{array}$ & Dependability & Credibility & ConQual score \\
\hline & & & & \\
\hline
\end{tabular}

Figure 3 ConQual summary of findings. GDM, gestational diabetes mellitus. and social adaptation experiences of women with GDM that can help identify challenges of glycaemic control and provide targeted care and interventions to improve maternal and child health.

\section{METHODS}

This review aims to synthesise experiences of women with GDM during the pregnancy. The research question will be appropriately answered by qualitative studies. The initial scope of the literature search provides recommendations for the proposed syntheses. The topic synthesis approach
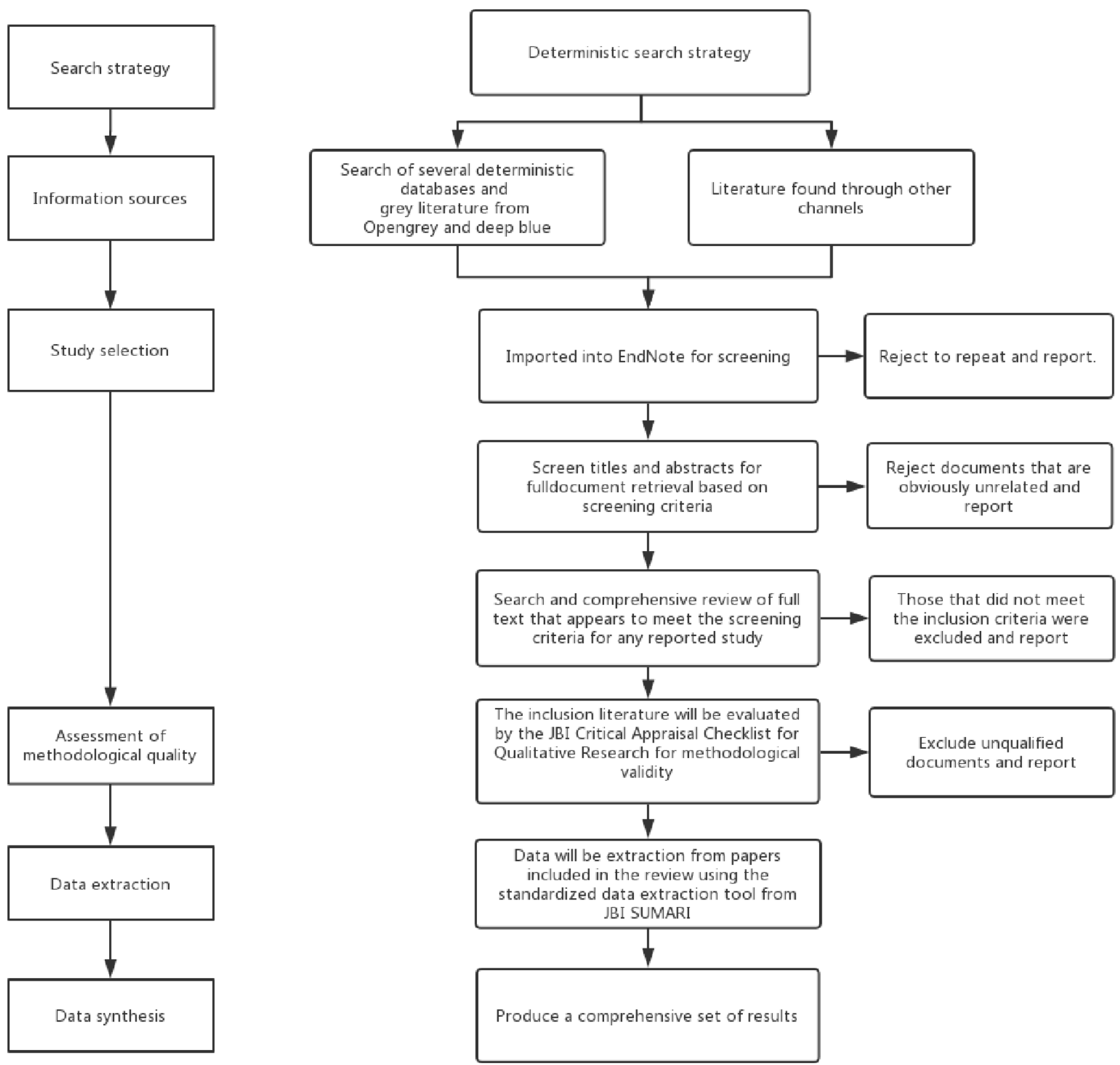

Figure 4 Search and selection process. online supplementary appendix 1: search strategy. JBI, Joanna Briggs Institute; SUMARI, System for the Unified Management, Assessment and Review of Information. 
Table 1 Summary of the Enhancing Transparency in Reporting the Synthesis of Qualitative Research statement

\begin{tabular}{cl}
\hline No & Item \\
\hline 1 & Aim \\
\hline 2 & Synthesis methodology \\
\hline 3 & Approach to searching \\
\hline 5 & Inclusion criteria \\
\hline 6 & Data sources \\
\hline 7 & Electronic search strategy \\
\hline 8 & Study screening methods \\
\hline 9 & Study characteristics \\
\hline 10 & Study selection results \\
\hline 11 & Rationale for appraisal \\
\hline 12 & Appraisal items \\
\hline 13 & Appraisal process \\
\hline 14 & Appraisal results \\
\hline 15 & Data extraction \\
\hline 16 & Software \\
\hline 18 & No of reviewers \\
\hline 19 & Coding \\
\hline 20 & Study comparison \\
\hline 21 & Derivation of themes \\
\hline & Quotations \\
\hline 17 & Synthesis output \\
\hline
\end{tabular}

involves the use of topic analysis techniques to identify key concepts or topics in primary studies, then form the title and complete the title registration in Joanna Briggs Institute (JBI) System and PROSPERO.

\section{Inclusion criteria}

Types of participants

This review will search and integrate studies of women with GDM during pregnancy. Searched studies will be eligible regardless of women's age and whether they have pregnancies for the first time or not.

\section{Phenomenon of interest}

This review will include studies that describe the experiences of women with GDM during the pregnancy.

\section{Context}

The context will consider in the experiences and feelings of women with GDM during the pregnancy.

\section{Types of studies}

The review will consider qualitative studies including, but not limited to, designs such as phenomenology, grounded theory, ethnography and feminist research.

\section{Patient and public involvement}

No patient will be involved in the design, planning and conception of this study.

\section{Search strategy}

The search strategy aims to find both published and grey literature. An initial limited search of PubMed will be conducted, following by an analysis of MeSH terms contained in the title and abstract, as well as the index terms used to describe the articles. This will inform the development of a search strategy which will be tailored for each database source. A second and complete search using all identified keywords and index entries will take place in all the relevant databases. Third, the reference list of all identified original studies will be examined for additional studies that may be relevant to this review. Previously published studies in English and Chinese will be considered for inclusion in the database. A full search strategy has been done (online supplementary appendix $1)$.

Information sources the Cochrane Library, Web of Science, JBI Database of Systematic Reviews and PsycINFO) and Chinese databases (ie, China Biology Medicine disc, China National Knowledge Infrastructure and VIP Database for Chinese Technical Periodicals). The search for grey literature will include OpenGrey and Deep Blue.

\section{Study selection}

All searched studies will be collated and uploaded into our software of EndNote X9. The duplicated studies will be removed. ${ }^{34}$ Two independent reviewers (JH and YW) will screen the title and abstract of the article based on the reviewer's inclusion criteria. Studies identified as potentially eligible or those without an abstract will have their full text retrieved and their details will be imported into the JBI System for the Unified Management, Assessment and Review of Information (JBI SUMARI). ${ }^{35}$ The two independent reviewers (JH and $\mathrm{YW}$ ) will retrieve and evaluate full texts of selected citations in detail which meet the inclusion criteria. Full texts that do not meet our inclusion criteria will be excluded, and excluding reasons will be attached as an appendix in the final systematic review report. ${ }^{34}$ Included studies will go through a critical screening process, and any differences between the two reviewers will be resolved through discussion. If no agreement is reached, a third reviewer (XC) will be involved.

\section{Assessment of methodological quality}

Qualitative papers selected for inclusion will be assessed by two independent reviewers (JH and $\mathrm{YW}$ ) according to the 10 items checklist of the JBI Qualitative Assessment and Review Instrument for methodological validity prior to inclusion in the review. ${ }^{36}$ This tool has been found to be more coherent and sensitive to effectiveness assessments than other commonly used tools. The evaluation content includes: methodology and philosophical perspective of the research, the research objectives, the methods used to collect data, analysis of data, the interpretation of results, if have statement for the influence of the researcher on the research, representativeness of the participants and the ethical and so on. All items were evaluated by using 
'yes', 'no' and 'unclear' to appraisal. An extract summary of the appraisal items is listed in figure 1 . The scores for these 10 items are similar to quantitative measures, with a score below or equal to 6 for a weak rating, 7-8 for a medium rating and 9-10 for a strong rating. References of the moderate rating or above will be included. Any disagreements that arise between the reviewers will be resolved through discussion by agreement or with a third reviewer (XC). Studies of the moderate rating or above will be included, extracted, synthesised from the data, and reflected in the results and conclusions of this system review. Low quality literature will be reported.

\section{Data extraction}

Two reviewers (JH and $\mathrm{YW}$ ) will independently extract data from papers by using the standardised data extraction tool from JBI SUMARI. The extracted data will include specific details (figure 2) about the study groups, context, culture, geographical location, study methods, the phenomena of interest relevant to the review question (ie, experiences of women with gestational diabetes during pregnancy in mental, physical and family life), and detail research objectives. Findings and their illustrations will be extracted and assigned a level of credibility.

\section{Data synthesis}

Qualitative research findings will be aggregated using JBI SUMARI with the meta-aggregation approach. There are three steps to integrate findings of the original study. First, individual findings will be appraised and will achieve one of three outcomes: unequivocal (evidence beyond reasonable doubt); credible (contains illustrations that may be challenged) or unsupported (when findings are not supported). Second, findings of all included studies will be extracted and categorised to create a set of categories representing meaningful similarities. Third, these similar categories are subjected to a synthesis in order to obtain a single comprehensive set of synthesised findings, which can be used as a foundation for evidence-based practice. For example, findings included 'husband's support' and 'mother in-law's support' that can be summarised as a category of 'family support'. The finding about 'support of nurses and midwives' will be summarised as a category for 'professional support'. Then, the two categories are subjected to create a set of synthetic results called 'social support'. If textual pooling is not possible, findings will be presented in a narrative form. Coding of findings, in time of the aggregation process to explore the influence, will be considered, about experiences of women with GDM during pregnancy. Two review authors will independently cluster identified findings, compare the generated categories and discuss discrepancies until reaching agreement. Finally, two authors of this study will work together to create a comprehensive set of synthesised findings.

\section{Assessing certainty in the finding}

The final findings will be graded according to the ConQual approach for establishing confidence in the output of research synthesis and presented in a summary of findings. ${ }^{37}$ The ConQual process was used to analyse the level of confidence or trust that exists in the value and level of evidence of each synthesised finding (figure 3). The figure will include the major elements of this systematic review and details on how the ConQual score is developed. The summary of findings will include the primary study title, phenomena of interest and context for the special review. The flow chart (figure 4) shows the whole protocol process, which will be completed by two reviewers independently, and then combined with our analysis.

\section{PRESENTING AND REPORTING THE REVIEW}

The resulting review will be reported in accordance with the 'Enhancing Transparency in Reporting the Synthesis of Qualitative Research' statement, ${ }^{38}$ which consists of 21 items and is appropriate for qualitative evidence synthesis (table 1).

\section{DISCUSSION}

This systematic review will discover women's experiences after being diagnosed with GDM during the pregnancy and integrate these findings for a comprehensive and in-depth understanding of their difficulties and needs. Specific personal experiences of women with GDM can inform professional healthcare and provide personalised care and education for these women. Improved care will promote maternal and child health, and therefore, reduce the medical burden.

This systematic review of qualitative evidence will be published in an open access, peer-reviewed and international journal for dissemination.

Correction notice This article has been corrected since it was published. Affilaition for 'Jing He' and corresponding authors have been updated.

AcknowledgmentsWe thank the librarian (Jin Chen) from Wuhan University for his guidance and assistance in retrieving the bibliographic database.

Contributors JH and YW contributed to the conception of the study. The manuscript protocol was drafted and finished the introduction by JH and YW. JH, YW and XC drafted and finished the Methods sections with assistance from YL. And the protocol was revised by JB. All the authors developed the search strategy and $\mathrm{JH}$ and $\mathrm{YW}$ will also independently screen the potential studies, extract data from the included studies, assess the risk of bias and complete the data synthesis. XC will arbitrate in cases of disagreement and ensure the absence of errors. All authors approved the publication of the protocol.

Funding This work was supported by the MOE (Ministry of Education in China) Project of Humanities and Social Sciences (grant number 17YJCZH113, 2017).

\section{Competing interests None declared.}

Patient consent for publication Not required.

Provenance and peer review Not commissioned; externally peer reviewed.

Open access This is an open access article distributed in accordance with the Creative Commons Attribution Non Commercial (CC BY-NC 4.0) license, which permits others to distribute, remix, adapt, build upon this work non-commercially, and license their derivative works on different terms, provided the original work is properly cited, appropriate credit is given, any changes made indicated, and the use is non-commercial. See: http://creativecommons.org/licenses/by-nc/4.0/.

ORCID iDs 
Jing He http://orcid.org/0000-0001-7654-1872

Yuchen Wang http://orcid.org/0000-0003-2433-4905

Xiaoli Chen http://orcid.org/0000-0001-6835-7768

\section{REFERENCES}

1 Moncrieff G. Gestational diabetes. Br J Midwifery 2018;26:506-13.

2 Mirghani Dirar A, Doupis J, Abdelhameed MD. Gestational diabetes from a to Z. World J Diabetes 2017;8:489-511.

3 Zhu Y, Zhang C. Prevalence of gestational diabetes and risk of progression to type 2 diabetes: a global perspective. Curr Diab Rep 2016;16:7.

4 Gao C, Sun X, Lu L, et al. Prevalence of gestational diabetes mellitus in mainland China: A systematic review and meta-analysis. $J$ Diabetes Investig 2019;10:154-62.

5 Thompson D, Berger H, Feig D, et al. Diabetes and pregnancy. Can J Diabetes 2013;37:S168-83.

6 Carolan-Olah MC. Educational and intervention programmes for gestational diabetes mellitus (GDM) management: an integrative review. Collegian 2016;23:103-14.

7 Veeraswamy S, Vijayam B, Gupta VK, et al. Gestational diabetes: the public health relevance and approach. Diabetes Res Clin Pract 2012;97:350-8

8 Farrar D, Fairley L, Santorelli G, et al. Association between hyperglycaemia and adverse perinatal outcomes in South Asian and white British women: analysis of data from the born in Bradford cohort. Lancet Diabetes Endocrinol 2015;3:795-804.

9 Hunter D, Burrows R, Mohide P, et al. Influence of maternal insulindependent diabetes mellitus on neonatal morbidity. Can Med Ass $J$ 1993;149:47-52.

10 Abokaf H, Shoham-Vardi I, Sergienko R, et al. In utero exposure to gestational diabetes mellitus and long term endocrine morbidity of the offspring. Diabetes Res Clin Pract 2018;144:231-5.

11 Carolan-Olah M, Duarte-Gardea M, Lechuga J. A critical review: early life nutrition and prenatal programming for adult disease. J Clin Nurs 2015;24:3716-29.

12 Umberto S, Barker DJ. Offspring of diabetic pregnancy: long-term outcomes. Semin Fetal Neonat M 2009;14:119-24.

13 Cremona A, O'Gorman C, Cotter A, et al. Effect of exercise modality on markers of insulin sensitivity and blood glucose control in pregnancies complicated with gestational diabetes mellitus: a systematic review. Obes Sci Pract 2018;4:455-67.

14 Sacks K, Friger M, Shoham-Vardi I, et al. Prenatal exposure to gestational diabetes mellitus as an independent risk factor for long-term neuropsychiatric morbidity of the offspring. Am J Obstet Gynecol 2016;215:380-1.

15 Kampmann Uet al. Gestational diabetes: a clinical update. World J Diabetes 2015;6:1065-72.

16 Bellamy L, Casas J-P, Hingorani AD, et al. Type 2 diabetes mellitus after gestational diabetes: a systematic review and meta-analysis. The Lancet 2009;373:1773-9.

17 Serlin D, Lash R. Diagnosis and management of gestational diabetes mellitus. Am Fam Physician 2009;80:1-6.

18 Poolsup N, Suksomboon N, Amin M. Effect of treatment of gestational diabetes mellitus: a systematic review and meta-analysis. PLoS One 2014;9:e92485.

$19 \mathrm{Xu} \mathrm{T}, \mathrm{He}$ Y, Dainelli L, et al. Healthcare interventions for the prevention and control of gestational diabetes mellitus in China: a scoping review. BMC Pregnancy Childbirth 2017;17.
20 Edwell J, Jack J, Testing GD. Narrative, and medical distrust. J Bioethic Inq 2017;14:53-63.

21 Lawson EJ, Rajaram S. A transformed pregnancy: the psychosocial consequences of gestational diabetes. Sociol Health IIIness 1994;16:536-62.

22 Carolan M. Women's experiences of gestational diabetes selfmanagement: a qualitative study. Midwifery 2013;29:637-45.

23 Jirojwong S, Brownhill S, Dahlen HG, et al. Going up, going down: the experience, control and management of gestational diabetes mellitus among Southeast Asian migrant women living in urban Australia. Health Promot J Austr 2017;28:123-31.

24 Dayyani I, Maindal H, Rowlands $\mathrm{G}$, et al. A qualitative study about the experiences of ethnic minority pregnant women with gestational diabetes. Scand J Caring Sci 2019.

25 Hui AL, Sevenhuysen G, Harvey D, et al. Stress and anxiety in women with gestational diabetes during dietary management. Diabetes Educ 2014;40:668-77.

26 Yee LM, McGuire JM, Taylor SM, et al. Social and Environmental Barriers to Nutrition Therapy for Diabetes Management Among Underserved Pregnant Women: A Qualitative Analysis. J Nutr Educ Behav 2016;48:170-80.

27 Devsam BU, Bogossian FE, Peacock AS. An interpretive review of women's experiences of gestational diabetes mellitus: Proposing a framework to enhance midwifery assessment. Women and Birth 2013;26:e69-76.10.1016/j.wombi.2012.12.003

28 Parsons J, Ismail K, Amiel S, et al. Perceptions among women with gestational diabetes. Qual Health Res 2014;24:575-85.

29 Dennison RA, Ward RJ, Griffin SJ, et al. Women's views on lifestyle changes to reduce the risk of developing type 2 diabetes after gestational diabetes: a systematic review, qualitative synthesis and recommendations for practice. Diabetic Medicine 2019;36:702-17.10.1111/dme.13926

30 Kieffer EC, Sinco B, Kim C. Health behaviors among women of reproductive age with and without a history of gestational diabetes mellitus. Diabetes Care 2006;29:1788-93.

31 Carolan-Olah MC. Educational and intervention programmes for gestational diabetes mellitus (GDM) management: an integrative review. Collegian 2015:S1322769615000062.

32 Duarte-Gardea MO, Gonzales-Pacheco DM, Reader DM, et al. Academy of nutrition and dietetics gestational diabetes evidence-based nutrition practice guideline. J Acad Nutr Diet 2018;118:1719-42.

$33 \mathrm{Kim} \mathrm{H}$, Kim S. Retraction: effects of an integrated self-management program on self-management, glycemic control, and maternal identity in women with gestational diabetes mellitus. J Korean Acad Nurs 2014;44:593-30.

34 Remington J, Winters K. Effectiveness of dietary inorganic nitrate for lowering blood pressure in hypertensive adults: a systematic review. JBI Database System Rev Implement Rep 2019;17:365-89.

35 Pearson A. Balancing the evidence: incorporating the synthesis of qualitative data into systematic reviews. JBI Reports 2004;2:45-64.

36 Lockwood C, Munn Z, Porritt K. Qualitative research synthesis: methodological guidance for systematic reviewers utilizing metaaggregation. Int J Evid Based Healthc 2015;13:179-87.

37 Munn Z, Porritt K, Lockwood C, et al. Establishing confidence in the output of qualitative research synthesis: the ConQual approach. BMC Med Res Methodol 2014;14:108.

38 Tong A, Flemming K, Mclnnes E, et al. Enhancing transparency in reporting the synthesis of qualitative research: ENTREQ. BMC Med Res Methodol 2012;12:181. 